\title{
Neuroepithelial stem cell marker nestin regulates the migration, invasion and growth of human gliomas
}

\author{
TOSHIYUKI ISHIWATA* , KIYOSHI TEDUKA* , TETSUSHI YAMAMOTO, \\ KIYOKO KAWAHARA, YOKO MATSUDA and ZENYA NAITO \\ Departments of Pathology and Integrative Oncological Pathology, \\ Nippon Medical School, Bunkyo-ku, Tokyo 113-8602, Japan
}

Received February 17, 2011; Accepted March 29, 2011

DOI: $10.3892 /$ or.2011.1267

\begin{abstract}
Nestin, a class VI intermediate filament protein, was originally described as a neuronal stem cell marker during central nervous system development. Nestin is expressed in gliomas, and its expression levels are higher in gliomas with high WHO histopathological classification grades than in those with low grades. In the present study, we examined whether nestin regulates the biological activities of human glioma cells. Immunohistochemically, the nestin expression patterns in 10 human glioblastoma patients were examined The expression levels of nestin in A172, a human high-grade glioma cell line, and KG-1-C, a human low-grade glioma cell line, were examined using real-time PCR, Western blot and immunofluorescence analyses. An expression vector carrying a short hairpin RNA targeting nestin was stably transfected into A172 (Sh) cells. The effects of decreased expression levels of nestin in Sh cells on cell growth, migration, invasion, adhesion to extracellular matrices and fibrillar actin expression on three-dimensional culture plates were examined. The nestin expression vector was transiently transfected into KG-1-C (Nes) cells, and the effects of the nestin overexpression on cell growth and migration were examined. Nestin was expressed in the cytoplasm of the glioblastoma cells in all cases examined. Sh cells showed marked decreases in the expression levels of nestin mRNA and protein, and the growth rate of Sh cells was lower than that of sham (Sc) cells. In contrast, the adhesion activity of Sh cells to types I and IV collagens, fibronectin and laminin was higher than that of Sc cells. Fibrillar actin was clearly detected at the periphery of colonies of Sh cells at the attachment sites on three-dimensional culture plates. The migration and invasion of Sh cells
\end{abstract}

Correspondence to: Dr Zenya Naito, Departments of Pathology and Integrative Oncological Pathology, Nippon Medical School, 1-1-5 Sendagi, Bunkyo-ku, Tokyo 113-8602, Japan

E-mail: naito@nms.ac.jp

*Contributed equally

Key words: nestin, glioblastoma, glioma, migration, invasion, cell proliferation were markedly inhibited compared with those of Sc cells. In contrast, the levels of nestin expression markedly increased in the Nes cells, which were transiently transfected with the nestin expression vector. The growth rate and motility of Nes cells were higher than those of the mock cells. In conclusion, nestin plays important roles in cell growth, migration, invasion and adhesion to extra-cellular matrices in glioma cells. Nestin may serve as a novel candidate for molecular-targeted therapy for gliomas, including glioblastomas.

\section{Introduction}

Gliomas are the most common primary neoplasms of the brain in adults. Gliomas exhibit the characteristics of glial cells, and different subtypes of gliomas are named according to the normal brain cell types that they most closely resemble. The main types of glioma are astrocytomas, oligodendrogliomas, ependymomas and mixed gliomas. Gliomas are further categorized according to their histopathological grades. Low-grade gliomas are not anaplastic and are associated with a favorable patient prognosis. In contrast, high-grade gliomas exhibit increased cellularity, nuclear atypia, mitotic activity, microvascular proliferation and necrosis, and this aggressive growth of the tumors results in a worse patient prognosis. Gliomas of the highest grade are named glioblastomas (1).

Surgical treatment is the main therapy, and radiotherapy and chemotherapy are performed as additional therapies for glioblastoma (2). Despite significant advances in treatment and intensive research, the prognosis for patients with glioblastoma remains poor. The five-year survival rate of patients with glioblastomas is approximately $3 \%$, and glioblastoma is known as one of the most malignant tumors in humans (3). Owing to their high growth rate, glioblastomas aggressively invade into adjacent normal brain tissues and are often surgically unresectable. Moreover, recurrent glioblastomas are resistant to conventional radiotherapy and chemotherapy. Many cell adhesion proteins, cytoplasmic proteins and growth factor receptors, including the neural cell adhesion molecule (NCAM), matrix metalloproteinase (MMP), integrin, CD44 and epidermal growth factor receptor (EGFR), play important roles in glioblastoma cell migration and invasion (4-6). These factors affect the intracellular states of tumor cells and induce their migration and invasion. Therefore, treatments targeting 
these molecules have been expected to inhibit glioblastoma cell migration and invasion, and improve the prognosis of glioblastoma patients (7-9).

Nestin is a member of the class VI intermediate filament (IF) proteins and was first described as a neuronal stem/progenitor cell marker (10). Nestin contains a short $\mathrm{N}$-terminus and an unusually long C-terminus and interacts with other IF proteins including vimentin and desmin, forming heterodimers; yet nestin cannot polymerize itself $(11,12)$. The long carboxy-terminus of nestin protrudes from its filament body and functions as a linker or a cross-bridge between IFs, microfilaments and microtubules $(10,13,14)$. Thus, nestin may coordinate the expression and functions of three components of the cytoskeleton including IFs, microfilaments and microtubules. Nestin has been implicated in the rapid proliferation of progenitor cells during neurogenesis (15). However, when precursor cells differentiate into neural or glial cell types, nestin expression has been shown to be down-regulated or to disappear $(16,17)$. An increased expression level of nestin has been demonstrated in various types of tumor cells, including central nervous system tumors, melanomas, gastrointestinal stromal tumors, prostate cancers, breast cancers and pancreatic cancers (18-21). In melanoma and prostate cancer, the expression of nestin correlates with cell migration, invasion and metastasis $(19,20)$.

Nestin has been detected in low-grade and high-grade gliomas, yet nestin immunostaining has been observed more frequently in high-grade gliomas than in low-grade gliomas such as pilocytic astrocytomas (22). The down-regulation of nestin in glioblastomas induces cell cycle arrest at the G1/S phase in the cells (23). However, the roles of nestin in gliomas have not been well clarified. In this study, we examined the expression pattern and the roles of nestin in glioma cells. We report here that expression of nestin correlates with cell growth, migration, invasion and adhesion to extracellular matrices (ECMs) in low- and high-grade gliomas.

\section{Materials and methods}

Materials. FuGene HD transfection reagent was purchased from Roche Diagnostics (Mannheim, Germany) and geneticin from Gibco BRL (Grand Island, NY). pBAsi-hU6 Neo DNA and pAcGFP1-N1 vectors were purchased from Takara Bio Inc. (Tokyo, Japan) and Clontech Laboratories, Inc. (Mountain View, CA), respectively. High-capacity cDNA reverse transcription kits, TaqMan gene expression master mix, and TaqMan gene expression assays for nestin (Hs00707120_s1) and 18S rRNA (Hs99999901_s1) were obtained from Applied Biosystems, Inc. (Carlsbad, CA). M-PER mammalian protein extraction reagent and Super Signal West Dura extended duration chemiluminescent substrates were from Pierce (Rockfold, IL). Coomassie blue (SERVA Blau G) was purchased from Serva Electrophoresis GmbH (Heidelberg, Germany) and the Immobilon P transfer membrane was from Millipore (Yonezawa, Japan). Goat polyclonal anti-nestin (G-20) and HRP-labeled donkey anti-goat IgG antibodies were purchased from Santa Cruz Biotechnology, Inc. (Santa Cruz, CA), and HRP-labeled goat anti-mouse IgG antibody was from American Qualex International, Inc. (San Clemente, CA). Poly-L-lysine-coated (35-mm) and multi-well glass- bottomed (35-mm) dishes were purchased from Matsunami Glass Industries, Ltd. (Osaka, Japan). Mouse monoclonal anti-nestin antibody (196908) and bovine fibronectin were obtained from R\&D Systems, Inc. (Westerville, OH). Alexa 488-labeled donkey anti-mouse IgG antibody was from Invitrogen Corporation (Carlsbad, CA), and Vectashield $\mathrm{H}-1200$ containing 4',6-diamidino-2-phenylindole-2 $\mathrm{HCl}$ (DAPI) was from Vector Laboratories, Inc. (Burlingame, CA). The WST- 8 cell counting kit was from Wako Pure Chemical Industries (Osaka, Japan). NanoCulture 96-well plates (NCP-L-MS) and NanoCulture medium (NCM-M) were purchased from Scivax Corporation (Kanagawa, Japan). Cell culture insert $(8.0-\mu \mathrm{m}$ pore size) and BioCoat Matrigel invasion chamber were obtained from Becton-Dickinson and Co. (Franklin Lakes, NJ). Diff-Quick staining kit was from Sysmex International Reagents Co., Ltd. (Hyogo, Japan), and bovine dermis-acid-solubilized type I collagen was from Koken Co., Ltd. (Tokyo, Japan). The Histofine Simple Stain MAX PO (M) kits were from Nichirei (Tokyo, Japan). All other chemicals and reagents were purchased from SigmaAldrich Corporation (St. Louis, MO).

Patients and tissues. Tissues from 10 patients with glioblastoma who received treatment at Nippon Medical School Hospital (Bunkyo-ku, Tokyo, Japan) from 2007 to 2009 were obtained for this study. The patients consisted of 7 males and 3 females, whose median age was 67 years (range, 18-82). Paraffin-embedded specimens were prepared for immunohistochemical analysis as described previously (18). This study was carried out in accordance with the principles embodied in the Declaration of Helsinki, 2008, and informed consent for the usage of tissues was obtained from each patient. Normal brain tissues were obtained from the surgical margin of glioblastomas from the same patients. Human fetal brain total RNA, which was pooled from 21 spontaneously aborted male/female Caucasian fetuses, was purchased from Takara Bio Inc. (Tokyo, Japan).

Glioma cell lines. A172, human glioblastoma, and KG-1-C, low-grade glioma, cell lines were obtained from Riken BioResource Center (Ibaraki, Japan). A172 cells were grown in RPMI-1640 medium containing $10 \%$ fetal bovine serum (FBS), and KG-1-C cells were cultured in DMEM containing $20 \% \mathrm{FBS}$ at $37^{\circ} \mathrm{C}$ in a humidified $5 \% \mathrm{CO}_{2}$ atmosphere.

Immunohistochemical analysis. Paraffin-embedded tissue sections $(3.5 \mu \mathrm{m})$ were immunostained using a Histofine Simple Stain Max PO (M) kit. After deparaffinization, endogenous peroxidase activity was blocked by incubation with $0.3 \%$ hydrogen peroxide in methanol for $30 \mathrm{~min}$, and the sections were incubated with the anti-nestin antibody (clone: $196908,1: 500$ ) for $16 \mathrm{~h}$ at $4^{\circ} \mathrm{C}$ using phosphate-buffered saline (PBS) containing $1 \%$ bovine serum albumin (BSA). Bound antibodies were detected using Simple Stain Max PO (M) reagents with diaminobenzidine-tetrahydrochloride (DAB) as the substrate, and the sections were counterstained with Mayer's hematoxylin. Negative control studies were performed by omitting the primary antibodies. The immunohistochemical result for nestin was evaluated as follows: when staining was noted in the cytoplasm of $>10 \%$ of the tumor cells, regardless 
of the intensity of staining, the cells were designated as being positive for nestin. Two investigators (T.I. and K.T.) independently evaluated all of the specimens in a blinded manner.

Real-time PCR analysis. Total RNA was extracted from the cells using a FastPure RNA kit. Total RNA ( $1 \mu \mathrm{g} / \mathrm{sample})$ was used for reverse transcription with a high-capacity cDNA reverse transcription kit following the manufacturer's protocol. Real-time PCR for nestin and 18S rRNA was performed using the StepOnePlus real-time PCR system (Applied Biosystems, Inc.) with specific primers and a TaqMan probe. PCR was carried out in $20-\mu 1$ reaction mixtures containing $10 \mu \mathrm{l}$ of $2 \mathrm{X}$ TaqMan gene expression master mix, $2 \mu \mathrm{l}$ of template cDNA, and $1 \mu \mathrm{l}$ of TaqMan gene expression assays. Cycling conditions were as follows: $20 \mathrm{sec}$ at $95^{\circ} \mathrm{C}$ and 40 cycles for $1 \mathrm{sec}$ at $95^{\circ} \mathrm{C}$ followed by $20 \mathrm{sec}$ at $60^{\circ} \mathrm{C}$. Real-time PCR results were expressed as target/18S rRNA, as an internal standard concentration ratio. Gene expression levels were measured in triplicate.

Western blot analysis. Protein was extracted from the cells in accordance with the protocol for the use of the M-PER mammalian protein extraction reagent. Lysates were centrifuged for $10 \mathrm{~min}$ at $13,000 \mathrm{rpm}$ to pellet cell debris, and the resulting supernatants were collected. The concentration of protein was measured by the Bradford method. The cleared protein lysates from the gliomas were subjected to sodium dodecyl sulfate-polyacrylamide gel electrophoresis (SDS-PAGE) under non-reducing conditions for nestin, and the separated proteins were transferred to Immobilon $\mathrm{P}$ transfer membranes. The membranes were immersed in 5\% skim milk in Tris-buffered saline containing $0.05 \%$ Tween-20 for $60 \mathrm{~min}$ at room temperature (RT), and incubated for $16 \mathrm{~h}$ at $4^{\circ} \mathrm{C}$ with a goat anti-nestin antibody (G-20; dilution, 1:500). The membranes were washed and incubated with HRP-labeled donkey anti-goat IgG (dilution, 1:4,000) for $60 \mathrm{~min}$. After washing, the blots were visualized using enhanced chemiluminescence and detected using a ChemiDoc XRS system (Bio-Rad Laboratories, Inc., Hercules, CA). To assess lane loading, the membranes were washed and reblotted with a mouse anti- $\beta$-actin antibody (dilution, 1:5,000).

Morphological analysis of gliomas. A172 and KG-1-C cells were cultured in 35-mm dishes in RPMI-1640 medium or DMEM containing 10 or $20 \%$ FBS for $48 \mathrm{~h}$. The cells were photographed using a Nikon Eclipse TE-2000-U photographic system (Nikon Insteck Co., Ltd., Tokyo, Japan) at x200 magnification.

Immunofluorescence analysis. A172 and KG-1-C cells (5x10 cells) were plated in $35-\mathrm{mm}$ glass-bottomed dishes and incubated for $72 \mathrm{~h}$ at $37^{\circ} \mathrm{C}$ in a humidified $5 \% \mathrm{CO}_{2}$ atmosphere. The cells were fixed in $4 \%$ paraformaldehyde solution for $15 \mathrm{~min}$ at RT. The fixed cells were treated with $50 \mathrm{mM}$ glycine for $5 \mathrm{~min}, 0.1 \%$ Triton $\mathrm{X}-100$ for $5 \mathrm{~min}$, and $10 \%$ donkey serum for $30 \mathrm{~min}$ at RT. The cells were then incubated with primary antibodies: incubation with a mouse anti-nestin antibody (clone, 196908; dilution, 1:50) overnight at $4^{\circ} \mathrm{C}$, followed by an Alexa 488-labeled anti-mouse IgG antibody (dilution, 1:1000). The dishes were subsequently mounted with Vectashield H-1200 containing DAPI. The nestin protein expression of cells was observed under a Digital Eclipse C1 TE2000-E microscope (Nikon Insteck Co., Ltd.) with a blue diode (excitation, $405 \mathrm{~nm}$; emission, 450/35 nm), an argon laser (excitation, $488 \mathrm{~nm}$; emission, 515/30 nm), and helium-neon lasers (excitation, $543 \mathrm{~nm}$; emission, 605/75 nm). Fluorescence images were acquired and analyzed using confocal microscope Digital Eclipse C1 control software EZ-C1 (Nikon Insteck Co., Ltd.). The confocal settings including laser power and detector sensitivity were unchanged during the acquisition of all images.

Construction of the expression vector for nestin short hairpin (sh)RNA and generation of stably nestin shRNA-expressing transfectants. To construct expression vectors for human nestin shRNA, a DNA fragment flanked by the BamHI and HindIII sites containing the sense target sequence for nestin (NM_006617; 5'-GAACAGGATCAGATGACAT-3'), the hairpin loop sequence (5'-TAGTGCTCCTGGTTG-3'), and the antisense target sequence were synthesized and inserted into the pBAsi-hU6 Neo DNA vector. Likewise, the scrambled sequence (5'-TCTTAATCGCGTATAAGGC-3') was used to construct the sham vectors that served as negative controls. A172 cells (1x10\%/well) were plated in 6-well plates and grown in $2 \mathrm{ml}$ of RPMI-1640 medium with $10 \% \mathrm{FBS}$. The nestin shRNA expression vector and sham vector were transfected into the cells using FuGENE HD transfection reagent, in accordance with the manufacturer's instructions. Briefly, $1 \times 10^{5}$ cells $/ \mathrm{ml}$ were transfected with $3 \mu \mathrm{g}$ of DNA using FuGene HD, and the cells were passaged and cultured with $600 \mu \mathrm{g} / \mathrm{ml}$ geneticin. To confirm the effective transfection of shRNA into the cells, cell lysates were collected, and nestin mRNA and protein expression levels were measured by realtime PCR and Western blot analyses, respectively.

Construction of the nestin expression vector and transient transfection into $K G-1-C$ cells. The full-length nestin cDNA fragment was ligated to the 3'-end of the human cytomegalovirus early promoter/enhancer in pAcGFP1-N1, a eukaryotic expression vector. The correct orientation of the insert was verified by DNA sequencing. The nestin expression and empty vectors were transfected using FuGENE HD transfection reagent. Seventy-two hours after the transfection, cell lysates were collected, and nestin mRNA and protein expression levels were measured by real-time PCR and Western blot analyses, respectively. The transiently transfected cells were used for the study.

Anchorage-dependent cell growth assays. To examine the growth rates of nestin shRNA-transfected A172 (Sh) cells and nestin-expression vector-transfected KG-1-C (Nes) cells, a non-radioactive cell proliferation assay was performed. The cells were plated at a density of $5 \times 10^{3}$ cells in 96-well plates with RPMI-1640 medium with 10\% FBS followed by incubation at $37^{\circ} \mathrm{C}$ in a humidified $5 \% \mathrm{CO}_{2}$ atmosphere for 24,48 and $72 \mathrm{~h}$. The cells were then incubated with WST- 8 cell counting reagent for $4 \mathrm{~h}$ at $37^{\circ} \mathrm{C}$ in a humidified $5 \% \quad \mathrm{CO}_{2}$ atmosphere, and the optical density of the culture solution in the plate was measured using an ELISA plate reader (Bio-Rad Laboratories) at $450 \mathrm{~nm}$. The experiments were performed in triplicate. 
Cell adhesion to extracellular matrices (ECMs). One hundred microliters each of bovine type I collagen, human type IV collagen, bovine fibronectin and murine laminin solutions, each at a concentration of $20 \mu \mathrm{g} / \mathrm{ml}$, was added into the wells of 96-well microplates; the microplates were then incubated at $4^{\circ} \mathrm{C}$ overnight. The residual culture area was blocked with $2 \% \mathrm{BSA}$ in PBS at $37^{\circ} \mathrm{C}$ in a humidified $5 \% \mathrm{CO}_{2}$ atmosphere for $2 \mathrm{~h}$. The cell suspension $(100 \mu \mathrm{l})$ was seeded at $2 \times 10^{4}$ cells/ well, and the cells were incubated at $37^{\circ} \mathrm{C}$ for $2 \mathrm{~h}$ in a humidified $5 \% \mathrm{CO}_{2}$ atmosphere. Non-adherent cells were removed by washing with serum-free medium. The relative number of attached cells was determined using a WST-8 cell counting kit. All assays were performed in triplicate.

Morphological analysis in three-dimensional (3D) cell cultures. The A172 cells were suspended in $0.1 \mathrm{ml}$ of NanoCulture medium containing 10\% FBS and plated on NanoCulture plates $\left(1 \times 10^{4}\right.$ cells $\left./ 0.1 \mathrm{ml}\right)$ for $3 \mathrm{D}$ cell culture. The plates were incubated at $37^{\circ} \mathrm{C}$ for $72 \mathrm{~h}$ in a humidified $5 \% \mathrm{CO}_{2}$ atmosphere. The cells were rinsed three times with PBS and then fixed with $4 \%$ paraformaldehyde solution for $20 \mathrm{~min}$ at RT. The cells were incubated with $50 \mathrm{mM}$ glycine for $10 \mathrm{~min}, 0.1 \%$ Triton X-100 for $5 \mathrm{~min}$, and $10 \%$ donkey serum for $30 \mathrm{~min}$ at RT. Next, cells were incubated with a mouse monoclonal antinestin antibody (clone, 196908; dilution, 1:50) at $4^{\circ} \mathrm{C}$ overnight and then washed with PBS. The cells were incubated with an Alexa 488-labeled donkey anti-mouse IgG antibody (1:1000 in dilution) and Alexa 568-labeled phalloidin (dilution, 1:50) for $60 \mathrm{~min}$ at RT. The cells were then mounted with Vectashield H-1200 containing DAPI. The nestin and fibrillar actin (F-actin) expression levels in glioma cells were observed under a Digital Eclipse C1 TE2000-E confocal microscope as described above. Images were collected at $0.5-\mu \mathrm{m}$ intervals with a laser to form a stack in the Z-axis, and were thus used to generate a 3D image using Volocity (Improvision, Coventry, UK).

Cell migration and invasion assays. To assess the effect of nestin on glioma cell migration, an in vitro migration assay using a modified Boyden chamber technique was carried out. Uncoated cell culture inserts $(8-\mu \mathrm{m}$ pore size and $6 \mathrm{~mm}$ in diameter) were used to assay cell migration following the manufacturer's instructions. Briefly, cells were suspended in $500 \mu \mathrm{l}$ of serum-free medium and placed onto the upper component of the inserts at a density of $1 \times 10^{5}$ cells. The lower compartment was filled with $750 \mu \mathrm{l}$ of medium containing $10 \% \mathrm{FBS}$, and the cells were incubated at $37^{\circ} \mathrm{C}$ in a humidified $5 \% \mathrm{CO}_{2}$ atmosphere. After $9 \mathrm{~h}$, the cells on the upper surface of the filter were carefully removed with a cotton swab. The cells that had migrated through the membrane to the lower surface of the filter were fixed and stained with a Diff-Quick staining kit and counted under a light microscope. The cell number on each membrane was counted in five high-power fields (x200). The cell invasion assay was performed using Matrigel-coated inserts, and the cells were counted as described above.

Single-cell movement assay. To confirm the effects of nestin on glioma cell migration, a single-cell movement assay was subsequently performed. A172 and KG-1-C cells (5,000 per well) were seeded onto a 4 -well glass-bottomed dish and incu- bated overnight. Cell movement was monitored for $24 \mathrm{~h}$ using a Digital Eclipse TE 2000-E motorized inverted microscope by capturing images every $5 \mathrm{~min}$. The total distance covered by individual cells within $24 \mathrm{~h}$ was determined using Metamorph software 7.6 (Universal Imaging Corp., Ltd., Buckinghamshire, UK).

Statistical analyses. All quantitative data are presented as mean \pm SE and were assessed using the Student's t-test and the Mann-Whitney test. Computations were performed using the Stat View J version 5.0 software package (SAS Institute, Inc., Cary, NC).

\section{Results}

Immunohistochemical analysis of nestin in the glioblastomas. Immunohistochemical analysis was initially carried out in order to characterize nestin expression in glioblastomas, which have the most malignant potential among gliomas. Nestin was localized in capillary endothelial cells in the non-neoplastic brain tissues obtained from the surgical margin of the glioblastomas (Fig. 1A, arrows and inset), but was not detected in the glial or neuronal cells. Immunohistochemically, nestin was strongly expressed in the cytoplasm of the glioblastoma cells, forming pseudopalisading necrosis and giant cells in all glioblastoma tissues examined (Fig. 1B and C, respectively). The nestin-expressing glioblastoma cells were diffusely localized in the central and peripheral lesions of the tissues.

Expression of nestin in the glioma cell lines. To examine the expression levels of nestin mRNA in glioma cells, real-time PCR analysis was performed in the A172 and KG-1-C cells, which originated from high-grade and low-grade glioma cells, respectively. Nestin mRNA was expressed in the A172 and KG-1-C cells, and its expression levels were higher in these cells than that in the fetal brain tissues (Fig. 2A). A strong band corresponding to the nestin protein was detected in the lanes for A172 and KG-1-C cells (Fig. 2B), and the protein was diffusely localized in the cytoplasm and the long processes of these cell lines (Fig. 2C).

Effects of the reduced expression level of nestin in A172 cells on cell growth and motility. To examine the roles of nestin in the growth, migration and invasion of glioma cells, we stably transfected A172 cells with the plasmid vector encoding shRNAs targeting nestin transcripts. Nestin shRNA reduced the expression level of nestin mRNA in the A172 (Sh) cells as compared with that in the sham $(\mathrm{Sc})$ cells $(\mathrm{p}<0.05)$ (Fig. 3A). Using these cells, an anchorage-dependent cell growth assay was performed to examine the effects of the reduced expression level of nestin on cell proliferation in the high-grade gliomas. The growth of Sh cells was inhibited compared with that of Sc cells, and the difference was statistically significant at 48 and $72 \mathrm{~h}(\mathrm{p}<0.001, \mathrm{p}<0.0005)$ (Fig. 3B). To examine the inhibitory effect of nestin on cell motility, cell movement was monitored for $24 \mathrm{~h}$ using a motorized inverted microscope by capturing images every $5 \mathrm{~min}$. The distance that the cells moved was measured, and the motility of Sh cells was found to be inhibited compared with that of Sc cells in the $24 \mathrm{~h}$ period $(\mathrm{p}<0.05)$ (Fig. 3C). 

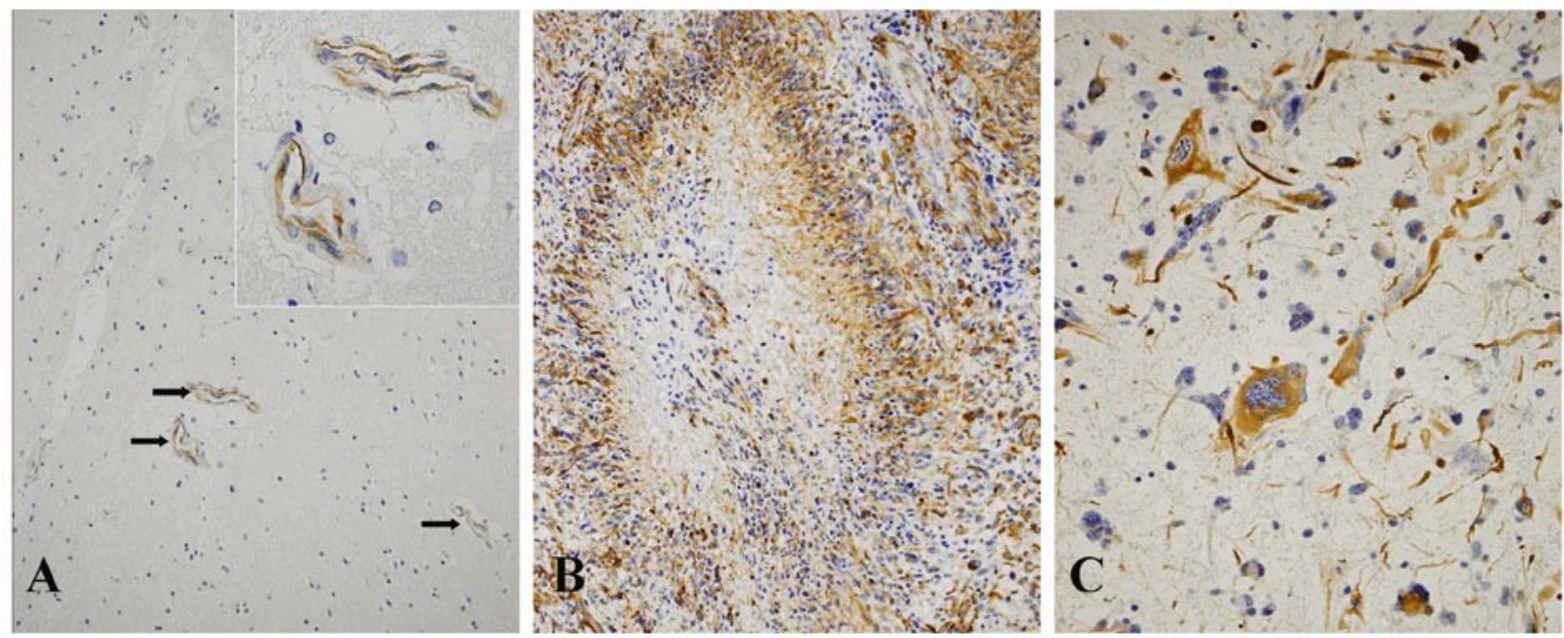

Figure 1. Immunohistochemical analysis of nestin in human glioblastoma tissues. Nestin protein was localized in capillary endothelial cells in non-neoplastic brain tissues obtained from the surgical margin of glioblastomas, but was not localized in glial cells or neurons (A). Strong nestin expression was detected in glioblastoma cells in pseudopalisading necrosis (B), but not in necrotic lesions. Nestin was also strongly expressed in giant cells in glioblastoma tissues (C). Immunohistochemistry: arrows, capillary endothelial cells. Original magnification: (A) x200; x600 (inset image); (B) x200; (C) x400.

A

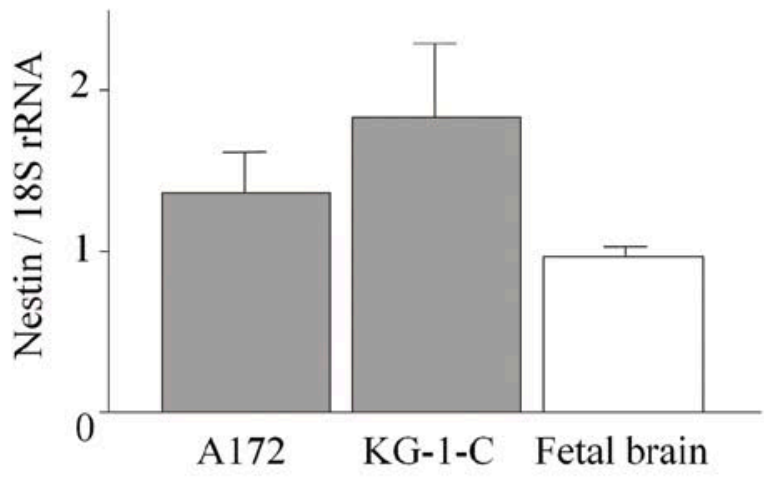

B
C

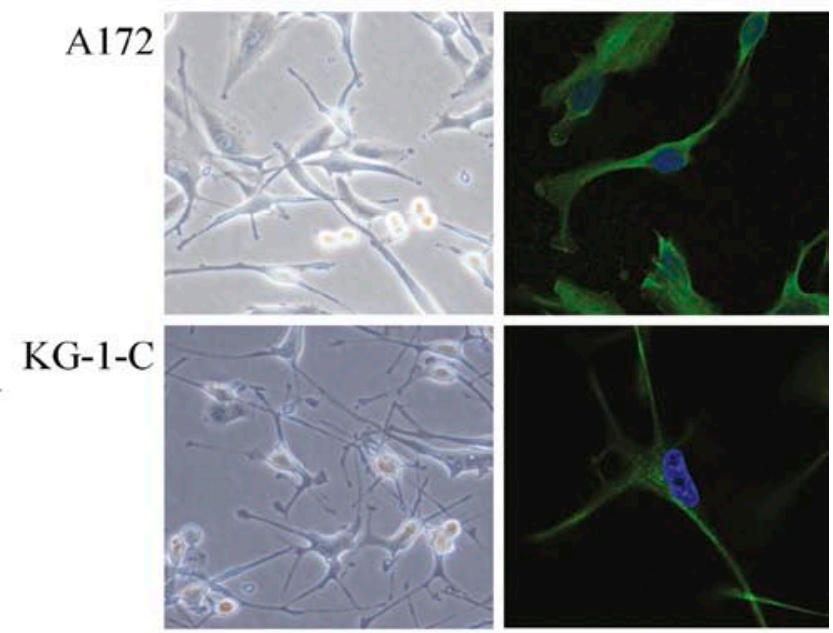

\section{Nestin}

$\beta$-actin

Figure 2. Expression of nestin in low- and high-grade glioma cell lines. Nestin mRNA was expressed in the A172 cells, KG-1-C cells and human fetal brain tissues. The expression levels of nestin in the KG-1-C cells and A172 cells were higher than that in the fetal brain, as determined by real-time PCR analysis (A). Nestin protein was detected in A172 and KG-1-C cells (B) and was strongly and diffusely expressed in the cytoplasm of these cell lines (C). (C) Left upper and lower panels, phase contrast images: original magnification, x200; right upper and lower panels, immunofluorescent images: original magnification, $\mathrm{x} 1000$.

Boyden chamber assay of cell migration and invasion. To confirm the effect of nestin on cell movement, we examined the migration and invasion of A172 cells using the modified Boyden chamber method. The number of A172 Sh cells that migrated was lower than that of A172 Sc after $9 \mathrm{~h}(\mathrm{p}<0.001)$ (Fig. 4A). A cell invasion assay was performed using the modified Boyden chamber method using Matrigel to coat the inner surface of inserts. The number of invading A172 Sh cells was lower than that of the invading A172 Sc cells after $9 \mathrm{~h}(\mathrm{p}<0.001)$ (Fig. 4B). These findings indicate that the decreased expression level of nestin inhibited the glioblastoma cell migration and invasion into the Matrigel.

Cell adhesion of stably nestin shRNA-transfected A172 cells to ECMs. We assayed the adhesion of nestin shRNAtransfected cells to major types of ECMs, including type I collagen, type IV collagen, fibronectin and laminin. All of the four ECM components enhanced cell adherence. Moreover, the degrees of adhesion of Sh cells to all ECMs were higher than those of Sc cells at a concentration of $20 \mu \mathrm{g} / \mathrm{ml}$ (Fig. 5A: 
A

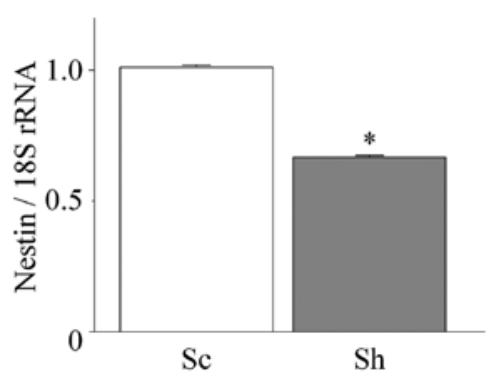

\section{B}

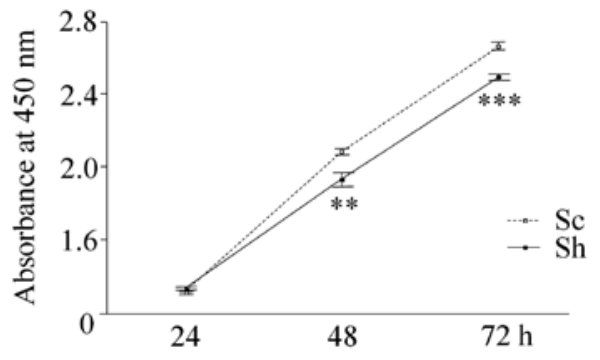

C

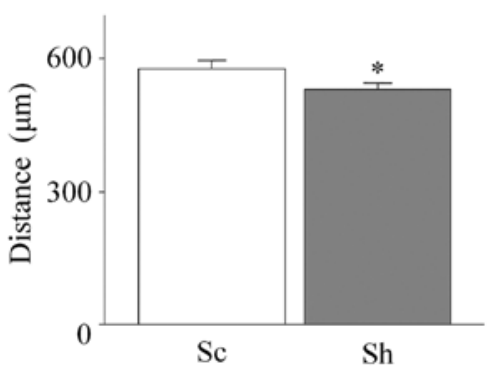

Figure 3. Effects of reduced expression of nestin in A172 cells on cell growth and motility. The nestin mRNA expression level was lower in the nestin shRNAtransfected A172 (Sh) cells than in the sham vector-transfected (Sc) cells (A). These cells were plated at a density of 5x10 3 in 96 -well plates with RPMI-1640 medium with $10 \%$ FBS and then incubated for 24,48 and $72 \mathrm{~h}$. The cells were then incubated with WST- 8 cell counting reagent. Cell growth rates were lower in the Sh cells than in the Sc cells (B). A172 cells (5,000 per well) were seeded onto a glass-bottomed dish and grown overnight. Cell movement was monitored for $24 \mathrm{~h}$ by capturing images every $5 \mathrm{~min}$. Cell motility was also lower in the Sh cells than in the Sc cells in $24 \mathrm{~h}$, using time-lapse analysis (C). ${ }^{*} \mathrm{p}<0.05,{ }^{* *} \mathrm{p}<0.001,{ }^{* * *} \mathrm{p}<0.0005$.

A
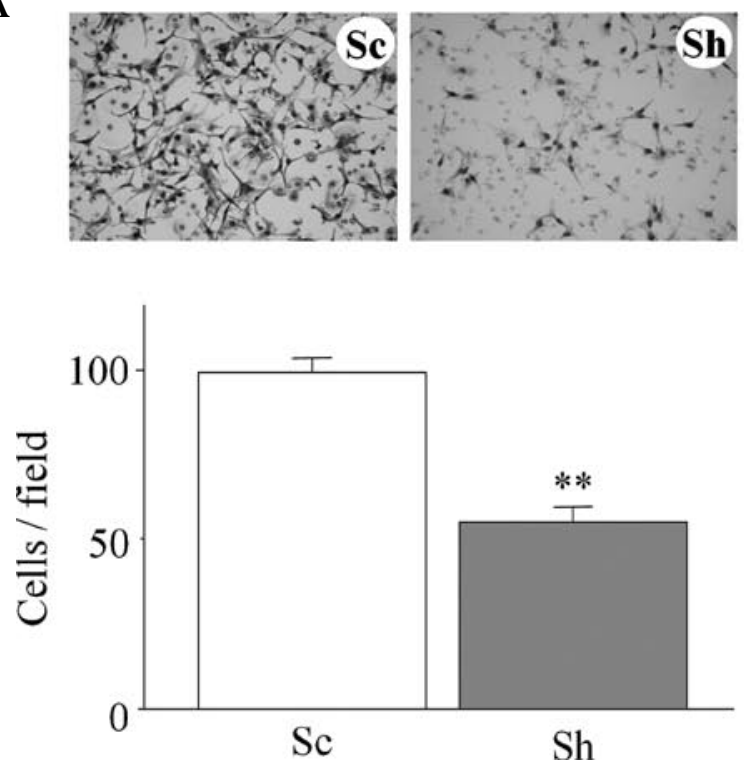

B
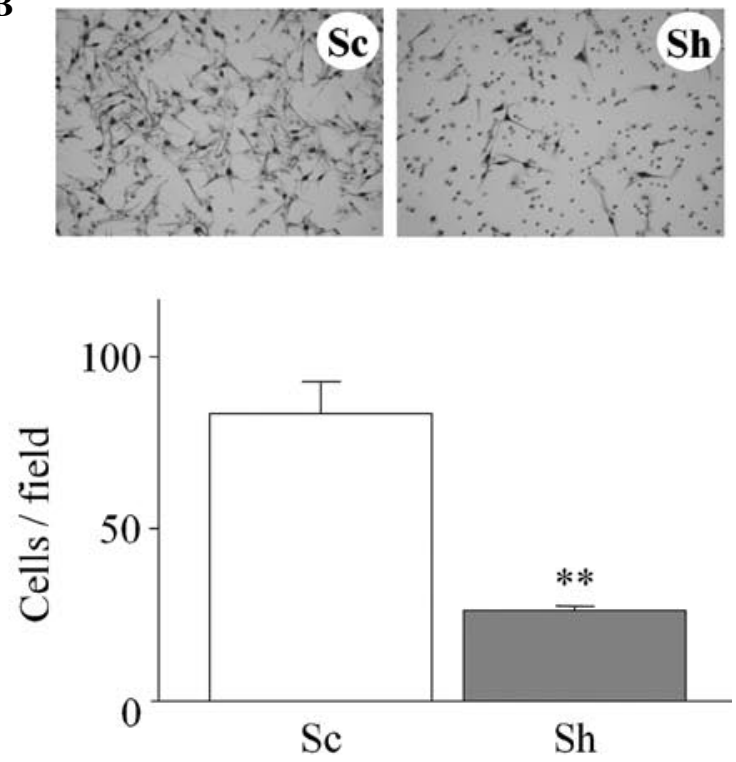

Figure 4. Boyden chamber assay for cell migration and invasion. Cells were suspended in $500 \mu 1$ of serum-free medium and placed onto the upper component of the inserts at a density of $1 \times 10^{5}$ cells. The lower compartment was filled with $750 \mu 1$ of a medium containing $10 \% \mathrm{FBS}$, and the cells were incubated at $37^{\circ} \mathrm{C}$ in a humidified 5\% $\mathrm{CO}_{2}$ atmosphere. After $9 \mathrm{~h}$, the cells that had migrated through the membrane to the lower surface of the filter were fixed, stained and counted under a light microscope. A cell invasion assay was performed using Matrigel-coated inserts. The number of A172 Sh cells that migrated was lower than that of A172 Sc cells (A). The number of invading A172 Sh cells was lower than that of A172 Sc cells after $9 \mathrm{~h}(\mathrm{~B}) .{ }^{* *} \mathrm{p}<0.001$.

solid bars, Sh cells; white bars, Sc cells). The adhesion activity of Sh cells to ECMs was statistically significantly higher than that of the Sc cells to type I collagen, type IV collagen and fibronectin (p<0.0005) (Fig. 5A).

Morphological analysis and expression patterns of nestin and $F$-actin in $3 D$ culture. To determine the mechanism under- lying the increased adhesion activity of Sh cells to ECMs, we performed 3D culture of Sh and Sc cells using NanoCulture plates, which have a specific microsquare pattern on the bottom of each plate that allows the formation of 3D spheroids (24). The distribution patterns of F-actin, one of the major cytoskeletal proteins, at the sites of cell attachment to the plates were examined in $3 \mathrm{D}$ culture and were analyzed using $3 \mathrm{D}$ 
A

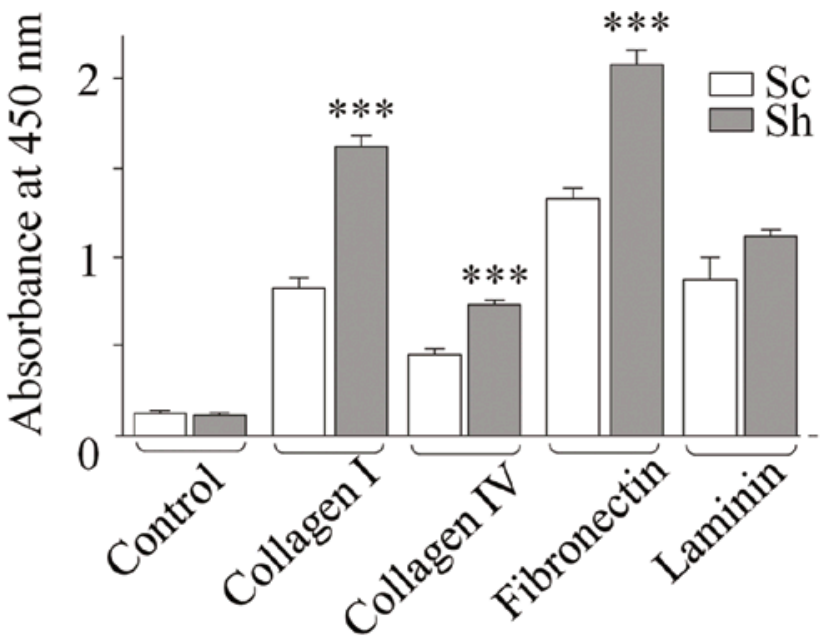

B
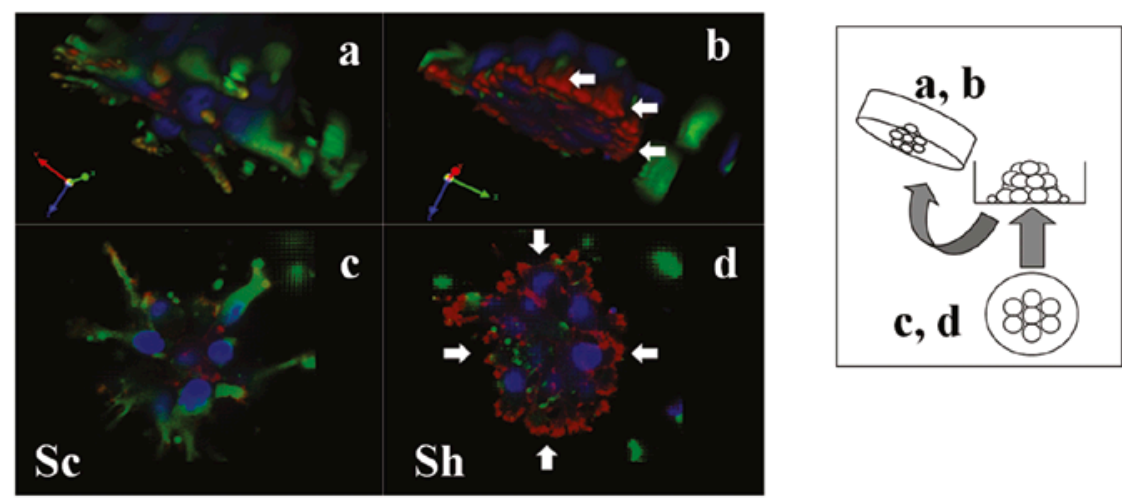

Figure 5. Adhesion activity to ECMs and F-actin expression of nestin shRNA-transfected A172 cells. Type I collagen, type IV collagen, fibronectin and laminin solutions (100 $\mu \mathrm{l} \mathrm{each)}$ at a concentration of $20 \mu \mathrm{g} / \mathrm{ml}$ were added to the wells, and cells were incubated at $37^{\circ} \mathrm{C}$ overnight. Then, $100 \mu 1$ of the cell suspension was seeded at $2 \times 10^{4}$ cells/well and incubated for $2 \mathrm{~h}$. Non-adherent cells were removed by washing, and the relative number of attached cells was determined using WST-8. The degrees of adhesion of Sh cells to all ECMs were higher than those of the sham cells at a concentration of $20 \mu \mathrm{g} / \mathrm{ml}$ (A). The A172 cells were suspended in NanoCulture medium containing 10\% FBS and plated on NanoCulture plates for 3D cell culture. Sc colonies appeared asteroid, whereas Sh cell colonies appeared nearly round (Ba and b). Sc cells showed a higher nestin expression level than Sh cells (Bc and d). Compared with Sc cells, Sh cells showed a markedly higher F-actin expression level particularly at the periphery of cell colonies at the sites of cell attachment (B, arrows). Mesh design in B shows the grids on the 3D-culture plates. ${ }^{* * *} \mathrm{p}<0.0005$; solid bars, Sh cells; white bars, Sc cells (A). Green, nestin; red, fibrillar actin; blue, nucleus; original magnification, $\mathrm{x} 1000(\mathrm{~B})$.

imaging software. The Sc cell colonies appeared asteroid, whereas the Sh cell colonies appeared nearly round (Fig. 5Ba and b). The Sc cells showed a higher nestin expression level than the Sh cells, and a strong expression was detected at the periphery of the colonies of the Sc cells (Fig. 5Bc and d). Compared with the Sc cells, the Sh cells showed a markedly higher F-actin expression level particularly at the periphery of the cell colonies on the sites of cell attachment (Fig. 5B, arrows).

Effect of the increased expression level of nestin in KG-1-C cells on cell growth and motility. To examine whether an increased expression level of nestin induces glioma cell growth and migration, nestin expression vectors were transiently transfected into low-grade glioma KG-1-C cells. The nestin mRNA expression level was high in nestin genetransfected KG-1-C cells (Nes, Fig. 6A), whereas it was low in mock cells, which were transfected with an empty vector $(\mathrm{p}<0.05)$. We compared the growth of KG-1-C cells that were transiently transfected with the nestin gene, with that of mock cells. Nestin gene-transfected cells exhibited higher growth rates than mock cells at 24,48 and $72 \mathrm{~h}(\mathrm{p}<0.05)$ (Fig. 6B).
Next, a single-cell movement assay was performed to examine whether an increased expression level of nestin induces cell movement as observed in A172 cells. The distance covered by nestin gene-transfected cells was longer than that covered by mock cells in $24 \mathrm{~h}(\mathrm{p}<0.05)$ (Fig. 6C).

\section{Discussion}

Nestin was first reported as a neuroepithelial stem cell marker in the fetal brain (10). Nestin-positive neuroepithelial stem cells are detected in the subventricular zone of the human adult brain and remain mitotically active throughout adulthood (25). Stem cells are defined as specific cells that have the capacities to self-renew and differentiate to all types of cells (26). Neuroepithelial stem cells can differentiate to neurons, oligodendrocytes and astrocytes (27). Several reports have indicated a close relationship between neuroepithelial stem cells and glioblastoma cells at their origin (28). Previously, nestin expression levels in gliomas were reported to correlate with their malignant potential (29). The nestin expression level is higher in high-grade gliomas than in low-grade gliomas. High levels of nestin expression in all glioblastoma 

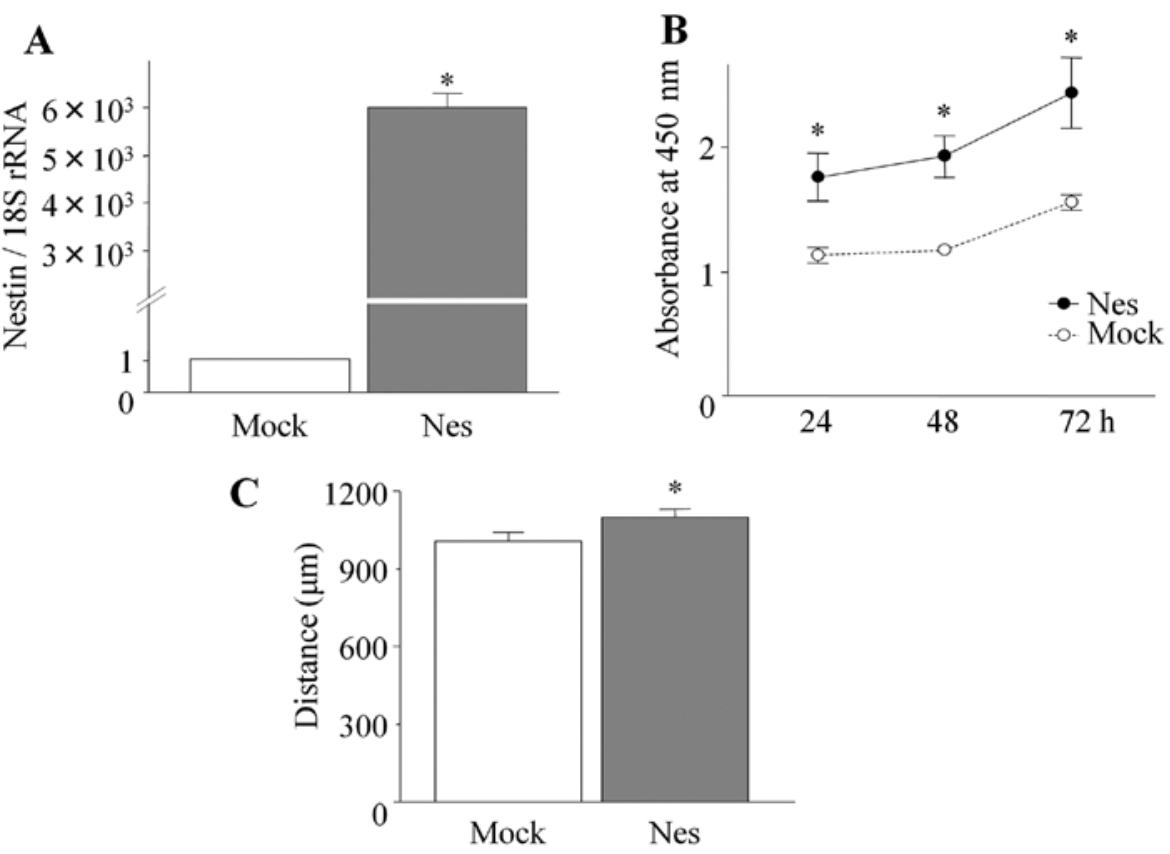

Figure 6. Effects of the increased expression level of nestin in KG-1-C cells on cell growth and motility. Nestin mRNA expression levels were high in nestin gene-transfected cells (Nes), whereas they were low in empty vector-transfected cells (mock), as determined by real-time PCR analysis (A). These cells were plated at a density of $5 \times 10^{3}$ in 96 -well plates in DMEM containing $20 \%$ FBS, incubated for 24,48 and 72 h, and further incubated with WST-8 cell counting reagent. The growth rates of Nes cells were higher than those of mock cells at 24,48 and 72 h (B). KG-1-C cells (5,000 per well) were seeded onto glass-bottomed dishes and incubated overnight. Cell movement was monitored for 24 h by capturing images every 5 min. The distances covered by nestin gene-transfected cells were longer than the distances covered by mock cells $(C)$. ${ }^{*} \mathrm{p}<0.05$.

cells suggest that glioblastomas are a transformed phenotype of neuroepithelial stem cells or dedifferentiated glial cells transformed to glioblastoma cells.

In the present study, nestin was not detected in glial cells of the non-neoplastic adult brain tissues obtained from the surgical margin of glioblastomas, but was strongly and diffusely expressed in all of the cases of glioblastoma, which has the highest grade among gliomas. The down-regulation of nestin inhibited high-grade glioma cell growth, migration and invasion to ECMs. Furthermore, nestin down-regulation in glioblastoma cells induced the accumulation of F-actin at the basal region of the cells and the tighter attachment to ECMs. In contrast, the overexpression of nestin in cells induced cell growth and cell motility. These findings suggest that nestin promotes glioma cell growth, migration and invasion. Alternatively, these findings suggest that glioblastoma cells take over part of stem cell functions such as cell proliferation and migration by overexpressing nestin.

In prostatic cancer, nestin expression correlates with cancer cell migration, invasion and metastasis, but not with cell growth (20). Consistent with our findings, the downregulation of nestin was found to induce G1/S cell cycle arrest in a rat glioblastoma cell line (23). To the best of our knowledge, this is the first report that the increased expression level of nestin induced the growth of tumor cells including gliomas. Low-grade gliomas often undergo transformation into a more advanced form with time, such as malignant transformation. This type of transformation has been reported to generally occur within 5 years in $\sim 50 \%$ of patients harboring these low-grade lesions (30). In the present study, the overexpression of nestin increased the grade of malignancy of low-grade gliomas. Therefore, nestin may have an important role in the acceleration of tumor progression.

In the present study, nestin shRNA-transfected cells showed markedly high expression of F-actin at the basal region of cell colonies in 3D culture. The expression of nestin was detected at the periphery of colonies in the sham cells, whereas it was not detected in nestin shRNA-transfected cells. Cells at the periphery of colonies are considered to have higher properties of migration, invasion and proliferation; therefore, the high nestin expression level observed in these cells in this study indicates that nestin correlates with these cell behaviors. Furthermore, the cytoskeletal reorganization of the actin filament is important in cell motility and is essential for most types of cell migration (31). Previous studies have shown that synemin, one of the large IF proteins structurally similar to nestin, directly regulates F-actin and $\alpha$-actinin in glioma cells $(32,33)$. Nestin is also reported to bind to microfilaments and microtubules at the C-terminus of the protein (12). These findings indicate that nestin directly regulates the expression levels and intracellular localization of F-actin in gliomas. The increased expression level of F-actin at the basal regions of the nestin shRNA-transfected cells may have contributed to their tighter attachments to ECMs and the inhibition of their migration and ECM invasion.

Microscopically, glioblastomas consist of pleomorphic cell types including multinucleated giant cells, astrocytic cells and spindle cells forming pseudopalisading necrosis. Nestin was diffusely expressed in all of the cell types in all of the glioblastoma cases examined, but not in astrocytes, oligodendrocytes or neurons in the non-neoplastic region. This indicates that the inhibition of nestin in glioblastoma reduces 
glioblastoma cell growth, migration and invasion without conferring side effects to normal glial cells and neurons in the brain.

In conclusion, this study showed that the down-regulation of nestin markedly inhibited high-grade glioma cell growth, migration and invasion, and induced adhesion of the cells to ECMs. In contrast, an increased expression level of nestin up-regulates low-grade glioma cell growth and migration. These results indicate a close relationship between neuroepithelial stem cells and glioblastoma cells at their origin, and nestin may be a novel molecular target in glioma therapy.

\section{Acknowledgements}

The authors thank Ms. Yoko Kawamoto, Mr. Takenori Fujii, and Ms. Taeko Suzuki (Department of Pathology, Integrative Oncological Pathology) for the excellent technical assistance. This study was supported by a grant-in-aid for scientific research to T. Ishiwata (C, no. 22591531) from the Japan Society for the Promotion of Science. This work was supported by grants-in-aid for young scientists from the Japan Society for the Promotion of Science to Y. Matsuda (A, no. 22689038) and T. Yamamoto (B, no. 22790675). This study was supported by grants (no. S0801035, to Z. Naito) from the Ministry of Education, Culture, Sports, Science, and Technology (MEXT), Japan.

\section{References}

1. Louis DN, Ohgaki H, Wiestler OD, et al: The 2007 WHO classification of tumours of the central nervous system. Acta Neuropathol 114: 97-109, 2007.

2. National Comprehensive Cancer Network (NCCN): Clinical practice guidelines in oncology: central nervous system cancers. J Natl Compr Cancer Netw 1: 2008.

3. Bondy ML, Scheurer ME, Malmer B, et al: Brain tumor epidemiology: consensus from the brain tumor epidemiology consortium. Cancer 113: 1953-1968, 2008.

4. Tews DS and Nissen A: Expression of adhesion factors and degrading proteins in primary and secondary glioblastomas and their precursor tumors. Invasion Metastasis 18: 271-284, 1998.

5. Rooprai HK, Vanmeter T, Panou C, et al: The role of integrin receptors in aspects of glioma invasion in vitro. Int $J$ Dev Neurosci 17: 613-623, 1999 .

6. Schunemann DP, Grivicich I, Regner A, et al: Glutamate promotes cell growth by EGFR signaling in the U-87MG human glioblastoma cell line. Pathol Oncol Res 16: 285-293, 2010.

7. Okamoto I, Kawano Y, Murakami D, et al: Proteolytic release of CD44 intracellular domain and its role in the CD44 signaling pathway. J Cell Biol 155: 755-762, 2001.

8. Okamoto I, Kawano Y, Tsuiki H, et al: CD44 cleavage induced by a membrane-associated metalloprotease plays a critical role in tumor cell migration. Oncogene 18: 1435-1446, 1999.

9. Okamoto I, Tsuiki H, Kenyon LC, et al: Proteolytic cleavage of the CD44 adhesion molecule in multiple human tumors. Am J Pathol 160: 441-447, 2002.

10. Lendahl U, Zimmerman LB and McKay RD: CNS stem cells express a new class of intermediate filament protein. Cell 60 $585-595,1990$

11. Steinert PM, Chou YH, Prahlad V, et al: A high molecular weight intermediate filament-associated protein in BHK-21 cells is nestin, a type VI intermediate filament protein. Limited co-assembly in vitro to form heteropolymers with type III vimentin and type IV alpha-internexin. J Biol Chem 274: 9881-9890, 1999.
12. Herrmann $\mathrm{H}$ and Aebi U: Intermediate filaments and their associates: multi-talented structural elements specifying cytoarchitecture and cytodynamics. Curr Opin Cell Biol 12: 79-90, 2000.

13. Hirokawa N, Glicksman MA and Willard MB: Organization of mammalian neurofilament polypeptides within the neuronal cytoskeleton. J Cell Biol 98: 1523-1536, 1984.

14. Hisanaga S and Hirokawa N: Structure of the peripheral domains of neurofilaments revealed by low angle rotary shadowing. J Mol Biol 202: 297-305, 1988.

15. Frederiksen K and McKay RD: Proliferation and differentiation of rat neuroepithelial precursor cells in vivo. J Neurosci 8: 1144-1151, 1988.

16. Zimmerman L, Parr B, Lendahl U, et al: Independent regulatory elements in the nestin gene direct transgene expression to neural stem cells or muscle precursors. Neuron 12: 11-24, 1994.

17. Loo DT, Althoen MC and Cotman CW: Down regulation of nestin by TGF-beta or serum in SFME cells accompanies differentiation into astrocytes. Neuroreport 5: 1585-1588, 1994.

18. Kawamoto M, Ishiwata T, Cho K, et al: Nestin expression correlates with nerve and retroperitoneal tissue invasion in pancreatic cancer. Hum Pathol 40: 189-198, 2009.

19. Florenes VA, Holm R, Myklebost O, Lendahl U and Fodstad O Expression of the neuroectodermal intermediate filament nestin in human melanomas. Cancer Res 54: 354-356, 1994.

20. Kleeberger W, Bova GS, Nielsen ME, et al: Roles for the stem cell associated intermediate filament Nestin in prostate cancer migration and metastasis. Cancer Res 67: 9199-9206, 2007.

21. Li H, Cherukuri P, Li N, et al: Nestin is expressed in the basal/ myoepithelial layer of the mammary gland and is a selective marker of basal epithelial breast tumors. Cancer Res 67: 501-510, 2007.

22. Ma YH, Mentlein R, Knerlich F, Kruse ML, Mehdorn HM and Held-Feindt J: Expression of stem cell markers in human astrocytomas of different WHO grades. J Neurooncol 86: 31-45, 2008.

23. Reimer R, Helmbold H, Szalay B, et al: Nestin modulates glucocorticoid receptor function by cytoplasmic anchoring. PLoS One 4: e6084, 2009

24. Matsuda Y, Ishiwata T, Kawamoto Y, et al: Morphological and cytoskeletal changes of pancreatic cancer cells in threedimensional spheroidal culture. Med Mol Morphol 43: 211-217, 2010.

25. Bernier PJ, Vinet J, Cossette $\mathrm{M}$ and Parent A: Characterization of the subventricular zone of the adult human brain: evidence for the involvement of Bcl-2. Neurosci Res 37: 67-78, 2000.

26. Tiedemann H, Asashima M, Grunz H and Knochel W: Pluripotent cells (stem cells) and their determination and differentiation in early vertebrate embryogenesis. Dev Growth Differ 43: 469-502, 2001.

27. Lee JC, Mayer-Proschel M and Rao MS: Gliogenesis in the central nervous system. Glia 30: 105-121, 2000.

28. Gil-Perotin S, Marin-Husstege M, Li J, et al: Loss of p53 induces changes in the behavior of subventricular zone cells: implication for the genesis of glial tumors. J Neurosci 26: 1107-1116, 2006.

29. Kitai R, Horita R, Sato K, et al: Nestin expression in astrocytic tumors delineates tumor infiltration. Brain Tumor Pathol 27: $17-21,2010$

30. Afra D, Osztie E, Sipos L and Vitanovics D: Preoperative history and postoperative survival of supratentorial low-grade astrocytomas. Br J Neurosurg 13: 299-305, 1999.

31. Yamazaki D, Kurisu S and Takenawa T: Regulation of cancer cell motility through actin reorganization. Cancer Sci 96: 379-386, 2005.

32. Bellin RM, Huiatt TW, Critchley DR and Robson RM: Synemin may function to directly link muscle cell intermediate filaments to both myofibrillar Z-lines and costameres. J Biol Chem 276: 32330-32337, 2001.

33. Pan Y, Jing R, Pitre A, Williams BJ and Skalli O: Intermediate filament protein synemin contributes to the migratory properties of astrocytoma cells by influencing the dynamics of the actin cytoskeleton. FASEB J 22: 3196-3206, 2008. 